\title{
Family-based cognitive behavioural therapy for obsessive-compulsive disorder with family accommodation: case report from Saudi Arabia
}

\author{
Yousra Alatiq* and Hind Alrshoud \\ Mental Health Division, King Abdulaziz Medical City, Riyadh, Saudi Arabia
}

\begin{abstract}
Family accommodation in obsessive-compulsive disorder (OCD) refers to a condition in which a family member assists or facilitates the patient with OCD in performing the compulsion, or provides assurance to minimize or reduce the anxiety level. This condition can be significantly disabling to the individual as well as to the family dynamic. In this case report, a mother of a 14-year-old female patient carried out almost all the compulsive behaviours and rituals for her child. Family-based cognitive behavioural therapy was offered to this case over a 4-month period with a successful treatment outcome. This result provides initial evidence that this type of intervention is suitable for patients from Saudi Arabia, a non-Western culture.
\end{abstract}

Key words: obsessive-compulsive disorder, family-based CBT, family accommodation, Saudi Arabia

\section{Introduction}

Obsessive-compulsive disorder (OCD) is an anxiety disorder characterized by intrusive thoughts and images (obsessions) and recurring, repetitive and ritualistic behaviours (compulsions) that are time consuming, significantly impair functioning and/or cause distress (American Psychiatric Association, 2013). Recent research has revealed a prevalence rate of 2-4\% among children and adolescents (Rapoport et al., 2000). In Saudi Arabia, the prevalence rate of mental health disorders has not been fully examined. However, in a recent study of adolescent girls, the prevalence of OCD was $13.7 \%$ (Atiq et al., 2017). The effect of OCD is not limited to the child alone; it includes all family members (Storch et al., 2009).

The literature on OCD in children reveals a useful construct of family interaction called family accommodation. Family accommodation is defined as 'the ways in which relatives, in particular parents, may assist in compulsive rituals, provide reassurance or modify their own routines to alleviate or avoid the distress experienced by the obsessive compulsive child' (Lebowitz et al., 2012). A recent meta-analysis suggests that studies of paediatric OCD do not

\footnotetext{
*Author for correspondence: Dr Y. Alatiq, Mental Health Division, King Abdulaziz Medical City, Riyadh, Saudia Arabia (email: yalatiq@gmail.com).
} 
usually report the level of accommodation by parents. However, when it has been reported, the prevalence is high (Lebowitz et al., 2012). One study found that $46 \%$ of parents participated in rituals or provided reassurance on a daily basis (Peris et al., 2008). Furthermore, higher levels of family accommodation were related to increased symptoms and impairment (Storch et al., 2007) and predicted poorer treatment outcomes (Albert et al., 2017).

Family accommodation is a useful framework to consider in child OCD. It may consist of varying degrees of involvement, from simple reassurance to active involvement. For example, family members may not only facilitate rituals and compulsions through reassurance but may also become over-involved and perform the rituals and compulsions themselves as a way of dealing with the problem. This high level of accommodation has been described as 'OCD by proxy' in two case reports of adult OCD in which the patients substituted another member of the family to carry out the compulsive behaviour (Vikas and Chandrasekaran, 2011; Ram and Kumar, 2014).

Treatment of OCD has developed significantly over the years. Numerous clinical trials have consistently shown improvement in patients with OCD using cognitive behavioural therapy (CBT) (Lack, 2012). A meta-analysis of the treatment of paediatric OCD shows a greater reduction of symptoms when using individual CBT (Abramowitz et al., 2005). Similar findings have been presented using family-based CBT (Iniesta-Sepúlveda et al., 2017). The literature on family accommodation suggests that lower accommodation after behavioural therapy leads to better outcomes (Lebowitz et al., 2012). Including the familial context in the conceptualization and treatment has been suggested to be a vital component for preventing dysfunctional patterns within the patient's environment that can cause relapse or symptom deterioration (Dummett, 2006; Thompson-Hollands et al., 2014).

In our clinical practice, we have encountered a number of OCD cases in which parents facilitated compulsive rituals by making modifications or offering assistance to their obsessive-compulsive child. However, in this case, we encountered an interactive dynamic in which the mother performed almost all the compulsive rituals for her child for years, suggesting a high level of family accommodation. This study seeks to document this case and examine the effect of family-based cognitive therapy as the main psychological intervention. To the best of our knowledge, no published studies have examined family-based CBT for the treatment of Saudi children and specifically for child OCD. Therefore, this paper contributes to filling the gap in this literature on the use of CBT in a non-Western culture and in a familybased format.

\section{Method}

\section{Case description}

A 14-year-old female patient presented to the Psychology Outpatient Clinic with a diagnosis of OCD and borderline intellectual functioning. The patient was in the 7 th grade and attending special education classes. She lived with both of her parents and seven siblings; she was the third to last child. She lived in a stable family environment with no family history of mental health disorders.

From approximately 3 to 7 years old, the patient had a complicated medical condition that required frequent emergency visits and hospital admissions. During this time, the patient began developing anxiety symptoms. These included sleep disturbances, fearfulness, 
irritability, school refusal, and separation anxiety symptoms. Furthermore, the mother's guilt and overprotection of her daughter's past and current medical condition had allowed her daughter to become fully dependent on her, as the mother reported. For example, the patient could not take care of her own needs and consistently needed to be showered, fed and dressed by her mother. This pattern of enmeshed interaction between the patient and her mother continued until the patient's current age.

The patient's anxiety symptoms escalated to a more complicated and rigid presentation. Certain compulsion behaviours began developing mainly around the interaction of the mother and the child on a daily basis. The majority of the patient's compulsions were specified in verbal demands; she could not perform a task unless the order was stated in the negative form. For example, if her mother asked her to brush her teeth, the patient would instruct the mother to say, 'Do not brush your teeth'. In addition, this statement would have to be reiterated in a specific pitch and manner; otherwise, the patient would not be able to carry out the task. The compulsive behaviours also included ordering her mother to walk in a specific way, mostly involving her moving backwards, then sideways, and then forwards to reach her destination. They also included the way her mother walked up and down the stairs. If these actions were not completed in the way the patient instructed, the patient would react with severe physical and verbal tantrums that could last an entire day. This rigidity was also observed with regard to the patient's own belongings and other elements in her environment, in which things had to be placed in a certain order. For example, the utensils on her food tray had to be organized in a certain way or she would be unable to eat.

The description and details of the compulsive behaviour were spontaneously reported by the patient and her mother, although the mother provided a more elaborate description due to the limited expressive ability of the patient. However, assessing the obsessions was more challenging. The patient was initially unable to report any form of obsessions or intrusions related to the compulsions. Efforts were made during assessments to encourage the patient to explore any underlying thoughts or beliefs that were associated with these compulsions. After a few attempts, the patient was able to identify obsessions related to an over-estimation of threat that needed to be controlled by the compulsions, such as, 'If things are not done in a certain way, something bad will happen'. The details of the obsessions and corresponding compulsions are described in Table 1.

During the clinical interview, it was revealed in the history that the patient had visited previous psychiatric clinics and had been given several inconsistent diagnoses, such as autism spectrum disorder, learning disability, attention deficit hyperactivity disorder, and separation anxiety. The patient was prescribed several medications based on these diagnoses and underwent laboratory investigations and neurological assessments. The last and current diagnosis was OCD with a referral for psychological intervention.

Observation during the clinical interview revealed the following: the patient was severely underweight and looked younger than her stated age. She appeared shy and made eye contact only with her mother. In addition, the patient's responses were verbalized slowly and in a low tone, and most of the time she gestured to her mother to answer for her. Another important observation was the pattern of interaction between the mother and the child, which resembled a pattern of over-attachment and over-protection. For instance, the mother and the child were sitting very close to each other and holding hands, and the mother complemented and apologized to the child when describing her symptoms; it appeared that she was overly concerned with hurting the child's feelings. Although the patient's verbal expression was 
Table 1. List of obsessions and compulsions

\begin{tabular}{ll}
\hline Obsessions & Compulsions \\
\hline $\begin{array}{l}\text { - Something very bad will happen to me, } \\
\text { my mother or our house }\end{array}$ & $\begin{array}{l}\text { Ordering her mother to do the following: } \\
\text { - Walking from the car to house in circle lines }\end{array}$ \\
- My mom will die & - Walking inside the house must be beside the walls \\
- I will die & (mother's shoulder must touch the walls) \\
- Something or someone will hurt & - Going up the stairs in the exact middle line \\
me/hurt mom & Verbally reversing any order stated to her (e.g. 'Do \\
- I will be sick again & your homework' to be 'Your homework, don't do it'; \\
& 'Put on your jacket' to be 'Your jacket, don't put it \\
& on') \\
- Putting the plates and utensils in very specific order \\
on the table or the tray \\
- Placing her belongings in specific and fixed places \\
(e.g. school bag to be beside the first step on the \\
stairs). \\
- Patting her hands 3-5 times each time she wears or \\
takes off something (e.g. jacket, shoes)
\end{tabular}

limited, it was clear that she was aware of her problem and that she was seeking help. In contrast, the mother looked exhausted and weary most of the time.

\section{Measures}

To measure the baseline level of severity and to establish therapeutic outcome measures, the following questionnaires were administered.

\section{The Symptom Checklist-90-R (SCL-90-R)}

This instrument is designed to screen for a vast range of psychological complications and symptoms of psychopathology. This multidimensional questionnaire consists of nine scales of primary symptom dimensions. However, only three relative dimensions are reported in this study: obsessive-compulsive traits, depression and anxiety. In addition, the scale contains three global indexes that assess overall psychological distress: the Global Severity Index (GSI), the Positive Symptom Total (PST) and the Positive Symptom Distress Index (PSDI). The cut-off score that indicates a need for psychological intervention is a T score above 60 (Derogatis, 1977). The Arabic version of the questionnaire has good psychometric properties (Albuhairi, 2005) and was used in this study.

\section{Generalized Anxiety Disorder-7 (GAD-7)}

This is a widely used self-report of anxiety symptoms. The questionnaire consists of seven items mainly designed for screening, diagnosing, and measuring generalized anxiety disorder. The total score on the GAD-7 for the seven items ranges from 0 to 21 (Spitzer et al., 2006). 


\section{The Patient Health Questionnaire-9 (PHQ-9)}

This is a widely used self-report screening for the presence and severity of depression. It consists of nine items with a total score ranging from 0 to 27 (Kroenke et al., 2001).

\section{Sheehan Disability Scale (SDS)}

This brief, 3-item self-administered questionnaire is designed to measure functional impairment in three main domains: work/school, social life, and family life. In each scale, patients rate from 0 to 10 the extent to which these domains are impaired by their symptoms (Sheehan, 1983).

\section{Ferrans and Powers' Quality of Life Index (QLI)}

This is used to assess satisfaction level in the main aspects of life. It consists of four domains: health and functioning, psychological/spiritual, social and economic, and family. The scale measures the level of satisfaction and evaluates it against the level of importance. The final overall score ranges from 0 to 30 , with a higher score suggesting higher quality of life (Ferrans and Powers, 1985).

\section{Treatment description}

After taking a full history of the patient's current and past difficulties, assessment of the cognitive and behavioural processes that may have played a role in triggering or maintaining the problem was conducted. A case formulation was then developed and shared with the patient and her mother. The therapy sessions consisted of sixteen sessions over 4 months held weekly. Detailed session-by-session content is listed in Table 2.

\section{Case formulation}

The case formulation was based on Padesky and Mooney's (1990) generic model, which is a framework for the way that cognitive, behavioural and environmental factors interact with each other to maintain current difficulties. We developed two interactive models for the child's and the mother's internal processes (see Fig. 1).

The formulation suggested that the complicated medical condition and frequent visits to emergency departments, with all the associated pain and suffering, were the primary triggers of the problem. However, different cognitive and behavioural processes in both the mother and the child played important and interactive roles in developing and maintaining the patient's anxiety and OCD.

Rituals and compulsive-like behaviour are considered part of normal development at certain ages (Evans et al., 1997). Certain levels of anxiety and fearfulness are also considered normal reactions to stress. Therefore, the child's feelings of anxiety or performance of rituals in response to the stress caused by her medical condition may have been normal at the early stage of the problem.

However, for the mother, there were different interpretations. She blamed herself for the possibility that she was somehow responsible for her sick child. She believed that her child was 'vulnerable' and would never be 'normal' like everyone else. These beliefs affected her emotions and motivated her overly caring and over-protective behaviour, which she displayed 
Table 2. Session-by-session content of the therapy

\begin{tabular}{|c|c|c|}
\hline Sessions & Session content & Homework assignment \\
\hline $1-2$ & $\begin{array}{l}\text { Assessment: } \\
\text { Presenting problem } \\
\text { Symptoms list and severity } \\
\text { Impairment of functioning } \\
\text { Family dynamic } \\
\text { Interpersonal history } \\
\text { Cognitive and behavioural processes }\end{array}$ & $\begin{array}{l}\text { Monitor and document all obsessions and } \\
\text { compulsion behaviours }\end{array}$ \\
\hline 3 & $\begin{array}{l}\text { Socialization to treatment: } \\
\text { Inform about diagnosis } \\
\text { Setting goals } \\
\text { Case formulation: } \\
\text { Develop and share case formulation } \\
\text { Psycheducation: } \\
\text { The disorder } \\
\text { The cognitive and behavioral model } \\
\text { The treatment approach }\end{array}$ & $\begin{array}{l}\text { Test case formulation through observation and } \\
\text { keeping record of own thoughts and behaviours for } \\
\text { both the mother and the child }\end{array}$ \\
\hline $4-10$ & $\begin{array}{l}\text { Behavioral approach: } \\
\text { Developed hierarchy of compulsion behaviours to target gradually; } \\
\text { Introduce exposure response prevention; } \\
\text { Teach effective coping skills when dealing with anxiety (e.g. breathing); } \\
\text { Praising and encouragement of progress }\end{array}$ & $\begin{array}{l}\text { Mother to practise gradually not responding to the } \\
\text { patient's demands (based on the identified } \\
\text { hierarchy of target behaviour) } \\
\text { Practise mindfulness breathing and other coping } \\
\text { techniques when anxiety arises (mother and child) }\end{array}$ \\
\hline $11-13$ & $\begin{array}{l}\text { Cognitive approach: } \\
\text { Examining underlying thoughts and interpretations; } \\
\text { Develop a more adaptive alterative through Socratic dialogue; } \\
\text { Introduce behavioural experiment to test alternative thoughts }\end{array}$ & $\begin{array}{l}\text { Test alternative thoughts and assumptions through } \\
\text { cognitive challenge and behavioural experiment } \\
\text { for the child }\end{array}$ \\
\hline $14-16$ & $\begin{array}{l}\text { Maintenance and relapse prevention } \\
\text { Review success in treatment and praising progress; } \\
\text { Identify possible trigger for relapse; } \\
\text { Develop effective action plan }\end{array}$ & $\begin{array}{l}\text { Practise appropriate emotional expression for the } \\
\text { child } \\
\text { Encourage focus on hobbies and expand social } \\
\text { network }\end{array}$ \\
\hline
\end{tabular}




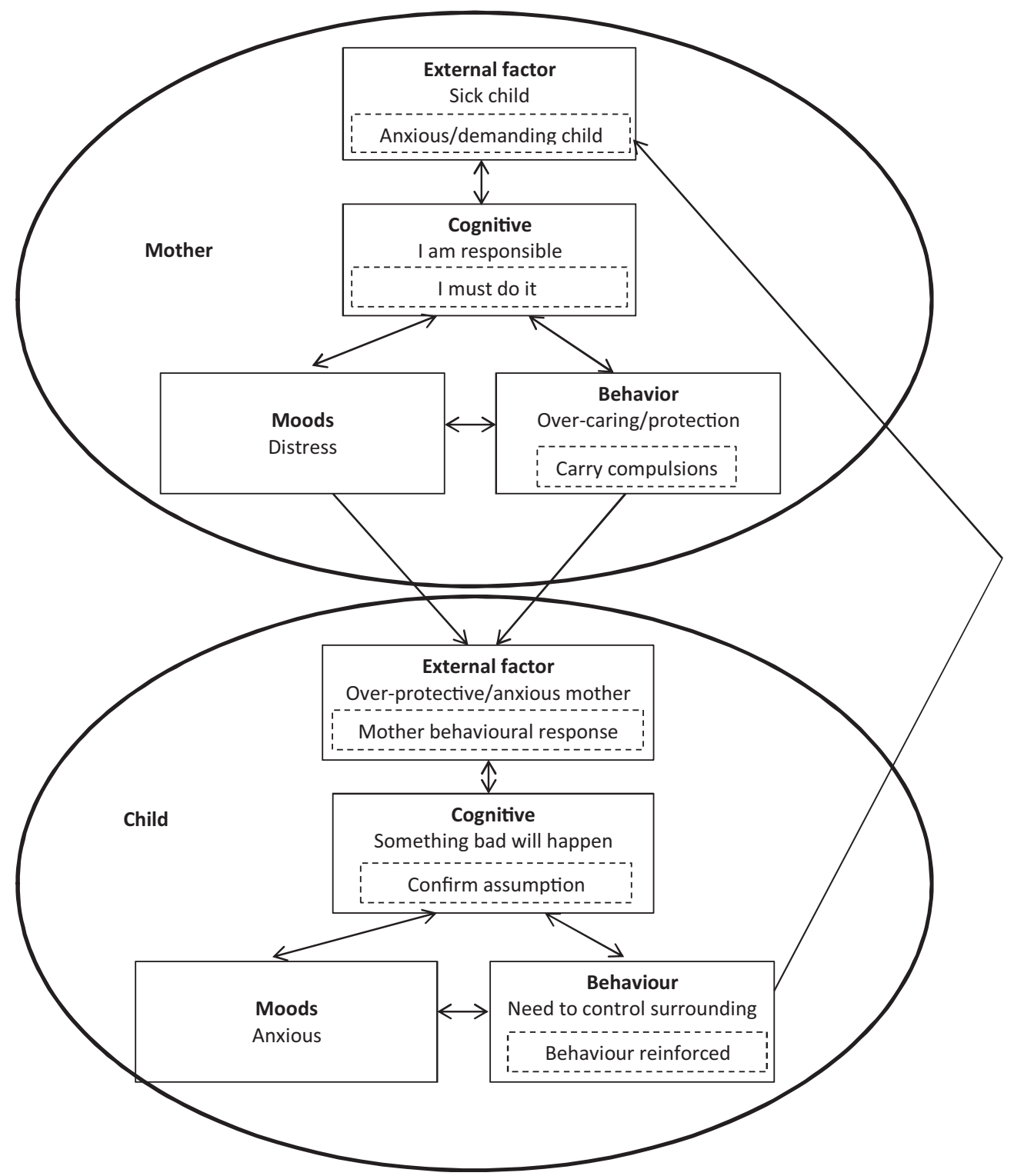

Figure 1. Interactive case formulation for OCD with family accommodation. The continuous line boxes represent the initial cycle of interaction, and the dotted line boxes represent a reactive response in the vicious cycle.

from early time. Moreover, as the child's anxiety increased, part of it was normal and part was reactive, as we will explain later. The mother's over-protective behaviours increased to a level at which she began performing compulsive behaviours for her child.

For a child who feels anxious and fearful, an anxious and over-protective mother may provide an additional environmental factor. This may occur through the interpretation that 
'danger is close' or simply by direct modelling of anxious behaviour or a conditioning process (Rachman, 1998). An assessment of the underlying beliefs related to compulsive behaviour revealed an over-estimation of threat; the patient stated that 'if things are not done in certain way, bad things will happen'. She later mentioned that her 'mother could die' or 'I will be sick again'.

In short, the current formulation of the interaction suggests that the anxious child believed that if things were not done in a certain way, 'bad things would happen'. The anxious mother picked this up and, because of her underlying sense of responsibility for her vulnerable child, would 'do things in certain way'. This led to a vicious cycle in which the child held the obsessions and the mother carried out the compulsions.

\section{Psychoeducation}

Comprehensive psychoeducation about OCD was a major part of the treatment. This included providing education about the nature of OCD and the cognitive behavioural model of the disorder to both the mother and the patient. Education was simplified for the child to match her level of understanding and comprehensive ability. This was followed by addressing the mother-daughter interaction pattern that maintained the problem.

\section{Behavioural approach}

Exposure and response prevention (ERP) is a major part of OCD treatment in general. It involves exposure to fear stimuli while refraining from or delaying the compulsive response. In collaboration with the mother, a detailed hierarchy of targeted compulsions was created (see Table 1 for the list of compulsions identified during the assessment). Because the mother was the primary individual performing the compulsions, she was the active client for this technique. Starting with the easiest and moving to the most challenging behaviours, the mother was asked to gradually stop responding to the patient's requests or tantrums (the feared stimuli). This was done after providing clear psychoeducation and rationale for this intervention.

Involvement of the patient in the behavioural approach was through, first, psychoeducation and rational of the technique (together with the mother); second, by using this exposure as an experiment to test the underlying thoughts 'what will happen if mom does not follow my demand'; third, through developing coping skills on how to deal with rising anxiety when the mother stop responding to her requests. These coping skills include breathing techniques as well as coping statements, e.g. 'this is a vicious cycle'.

\section{Cognitive approach}

This approach focused on assessing and examining the content of the thoughts by discussing the pattern of misinterpretations and underlying beliefs. The patient and her mother were asked to monitor their thoughts during the week and keep a record to be discussed in sessions. Working towards developing more adaptive beliefs was accomplished though Socratic questioning, mainly for the mother, and through behavioural experiments for the child. For example, the child was asked to test whether misplacing her table set could lead to bad things happening. Of course, this was done after ensuring that the patient understood the rationale of the experiment and after working to develop effective coping skills, such as breathing exercises. 
Table 3. Pre- and post-assessment measures

\begin{tabular}{lll}
\hline Subscales & Pre & Post \\
\hline The Symptom Checklist (SCL-90- R) & & \\
Obsessive-compulsive (O-C) & $2.70(\mathrm{~T}=66)$ & $1.80(\mathrm{~T}=50)$ \\
Depression (DEP) & $2.46(\mathrm{~T}=55)$ & $1.31(\mathrm{~T}=38)$ \\
Anxiety (ANX) & $2.10(\mathrm{~T}=51)$ & $1.10(\mathrm{~T}=35)$ \\
Global Severity Index (GSI) & $2.24(\mathrm{~T}=57)$ & $1.28(\mathrm{~T}=37)$ \\
Positive Symptom Total (PST) & $83(\mathrm{~T}=57)$ & $74(\mathrm{~T}=52)$ \\
Positive Symptom Distress Index (PSDI) & $2.43(\mathrm{~T}=49)$ & $1.56(\mathrm{~T}=33)$ \\
The Patient Health Questionnaire (PHQ-9) & 14 & 7 \\
Generalized Anxiety Disorder (GAD-7) & 14 & 6 \\
Sheehan Disability Scale (SDS) & 23 & 10 \\
Ferrans and Powers' Quality of Life Index (QLI) & & \\
Health and Functioning (HF) & 13.00 & 16.83 \\
Social and Economic (SE) & 12.13 & 19.36 \\
Psychological and Spiritual (PS) & 7.20 & 16.43 \\
Family (FT) & 26.38 & 29.38 \\
Overall score & 13.88 & 19.00 \\
\hline
\end{tabular}

$\mathrm{T}=\mathrm{t}$ score.

\section{Maintenance and relapse prevention}

In this end part of the treatment, we focused on maintaining improvement through continued practising of techniques and praising progress. We also focus on termination issues such as the identification of the treatment's successful aspects, identification of triggers and other factors that might suggest relapse, such as the mother's own anxiety, the child's limited emotional expression and social interaction, and finally develop a relapse prevention action plan.

\section{Results}

At the end of the therapy, all post-assessments revealed a significant decrease in the severity of the symptoms. On the SCL, the patient's score decreased on the obsessive-compulsive traits subscale (pre $=66$, post $=50$ ), the depression subscale $($ pre $=55$, post $=38$ ), and the anxiety subscale (pre $=51$, post $=35$ ). All results for these three items were above the cutoff score pre-therapy and significantly decreased to average scores post-therapy (see Table 3). In addition, all three global indexes, the GSI, the PST and the PSDI, showed a reduction in symptoms.

For the GAD-7 and PHQ, an important decrease in scores was observed as well. The patient's scores were pre $=14$ and post $=6$ on the GAD-7, and pre $=14$ and post $=7$ on the PHQ, indicating that the initial levels of moderate to severe anxiety and depression decreased to mild symptoms. On the SDS, the patient's pre-score was 23 , which indicates a high level of functional impairment, whereas the post-score was 10, which indicates a low level of functional impairment. On the QLI, the patient scored pre $=13.88$ and post $=19$, which again both reflect a better level of quality of life.

The assessment results indicate a significant improvement, which is in line with the clinical observation and report by the patient and her mother in the last sessions. There 
was a significant decrease in obsessional thoughts and a complete absence of tantrums. The compulsive behaviours were significantly reduced. The patient no longer asked the mother for any of the behavioural orders that were initially stated. The negatively stated verbal commands were also reduced significantly (distanced duration and less intensity). The mother reported that she had become more aware of her attitude of perceiving her daughter as 'vulnerable', which did not allow her daughter to practise independent skills. Concurrently, the patient's success in enduring the anxiety caused by the re-arrangement or modification of items reinforced the mother's awareness of her enabling role in the dynamic. In addition, the mother reported increase independent social activity by her daughter (she was able to enrol in social activities without mother's company, and would visit and sleep over in cousins' house without mother's presence), which is a significant improvement for both the mother and the child.

Finally, the patient and her mother were given an open appointment to come to the clinic whenever necessary. They were encouraged to continue to monitor symptoms and underlying tendencies and to seek help if an old pattern or symptoms returned, or if they simply needed a booster session to reinforce new skills.

\section{Discussion}

In this study, we report on family-based cognitive therapy for a case of OCD with a high level of family accommodation in which the mother of a 14-year-old patient performed all compulsive behaviours as demanded by the patient. We believe that reporting this case will add to the limited literature on this condition, especially in the Saudi cultural context.

In some studies of family accommodation, the parents were used as coaches or co-therapists to facilitate exposure and to reduce accommodation (Lebowitz et al., 2012). However, in our case, the interaction between the child and her mother was the main target of treatment and the focus of attention. Therefore, the case formulation developed for this patient was an interactive format that incorporated the interaction between the mother and the child's own processes. We developed this conceptualization because it was difficult to consider each component in isolation. Only when we considered the interactive pattern did the dynamic begin to make sense for the therapist as well as the patient and her mother. Consistent with this model, it has been theorized that symptoms of OCD and family dysfunction operate in a cyclical manner and mutually influence each other (Waters and Barrett, 2000).

The importance of developing a systemic cognitive behavioural formulation has been well emphasized (Dummett, 2006). The argument is that we cannot treat young children without considering all possible factors, including attachment patterns, the wider family system and cultural variables. Dummett developed a generic case formulation reflecting this approach. Although this formulation is quite comprehensive and simple to use, we have not used it for a number of reasons. First, it emphasizes the child as central to the formulation, whereas in our case, the interactive dynamic was the heart of the problem.

Second, Dummett's formulation was based on the Beakian model that emphasizes the three levels of cognition: automatic thoughts, assumptions and core beliefs. From our personal experience working with Saudi clients, and for this client in particular who had learning difficulties, this level of specification is not particularly useful and may confuse clients who have no previous knowledge of or experience with CBT. It makes more sense to consider all elements of thoughts and assumptions as 'cognition'. The therapist can remain flexible in using different techniques to address different thought elements. 
Third, the model emphasizes wider contextual and cultural factors that may have played a role in this problem. In our case, the cultural and wider context was not evaluated as the primary factor. Although one should never ignore the assessment of such important factors, in our case study, the cultural context and the wider family system were not the primary factors in the development and maintenance of the problem. We viewed this case as simply a stress reaction in light of an over-protective mother.

A CBT approach was selected for this case because of encouraging research findings on OCD for children (Abramowitz et al., 2005), in addition to a family-based approach (Barrett et al., 2004). However, the model used to understand this case and develop a formulation and treatment plan was not based on OCD-specific models such as Salkovskis's (1997) OCD cognitive model but rather a more generic model (Padesky and Mooney, 1990). This decision was made because most OCD-specific models focus on the meaning and interpretations given to the intrusions. In our case, the patient had limited verbal expression and comprehension ability; therefore, an in-depth analysis and interpretation of meaning beyond 'something bad will happen' or 'I will be sick again' were difficult to conduct.

In addition, although a disorder-specific model has strong evidence based on its original cultural context, evidence of its effectiveness is lacking in the Saudi community. A more transdiagnostic approach to case formulation and treatment plans has preliminary evidence that is suitable for treating emotional disorders in adult Saudi clients (Atiq, 2014). The transdiagnostic approach focuses on cognitive and behavioural processes that have been found to be common across disorders. Therefore, it provides the flexibility needed to assess and conceptualize relatively uncommon or complicated conditions such as the case at hand.

Given that neither the client nor her mother had any prior knowledge of or exposure to psychological intervention or CBT, we were vigilant in simplifying the case formulation and the rationale for the interventions and techniques used as much as possible. This approach emphasizes the role of psychoeducation in the treatment, which forms the foundation for any possible change. This is particularly important in a culture where knowledge and understanding of cognitive behavioural therapy might not be as common as in a Western culture, where CBT was originally founded.

The treatment was conducted in 16 sessions over 4 months and revealed a significant reduction in objective and subjective measures. These findings provide initial evidence that family-based CBT (which focuses on the interaction between the patient and the caregiver) is beneficial for the treatment of child OCD with a high level of family accommodation. The findings also add to the limited literature that this approach is suitable in a non-Western society and is particularly suitable for Saudi culture.

\section{Main points}

Family-based CBT is effective in treating child OCD with high level of family accommodation as measured by case report from Saudi Arabia.

\section{Acknowledgment}

The authors thank the patient and her mother who agreed for this case report to be published. 


\section{Conflicts of interest}

None.

\section{Ethical statement}

This case study was reviewed and approved by the IRB at King Abdullah International Medical Research Centre (KAIMRC).

\section{Financial support}

There was no financial support for this study.

\section{Recommended follow-up reading}

Marien WE, Storch EA, Geffken GR, Murphy TK (2009). Intensive family-based cognitivebehavioral therapy for pediatric obsessive-compulsive disorder: applications for treatment of medication partial or nonresponders. Cognitive and Behavioral Practice 16 (3).

Lebowitz ER, Bloch M (2012). Family accommodation in pediatric obsessive compulsive disorder. Expert Review of Neurotherapeutics 12, 229-238.

\section{References}

Abramowitz JS, Whiteside SP, Deacon BJ (2005). The effectiveness of treatment for pediatric obsessive-compulsive disorder: a meta-analysis. Behavior Therapy 36, 55-63.

Albert U, Baffa A, Maina G (2017). Family accommodation in adult obsessive-compulsive disorder: clinical perspectives. Psychology Research and Behavior Management 10, 293-304.

Albuhairi AA (2005). SCL-90_R, Arabic version. Asuod, Eyget: Counseling and Educational Center.

American Psychiatric Association (2013). Diagnostic and Statistical Manual of Mental Disorders, DSM-5. Washington, DC: American Psychiatric Association.

Atiq Y (2014). Transdiagnostic cognitive behavioural therapy (CBT): case reports from Saudi Arabia. Cognitive Behaviour Therapist 7, 1-12.

Atiq Y, Alshalan M, Almodayfer O (2017). Prevelance of psychiatric disorders among Saudi female adolescent girls in Riyadh City High School. The Arab Journal of Psychiatry 28, 160-168.

Barrett P, Healy-Farrell L, March JS (2004). Cognitive-behavioral family treatment of childhood obsessive-compulsive disorder: a controlled trial. Journal of the American Academy of Child and Adolescent Psychiatry 43, 46-62.

Derogatis LR (1977). SCL-90-R Administration Scoring and Procedures Manual I. Baltimore: Clinical Psychometric Research.

Dummett N (2006). Processes for systemic cognitive behavioural therapy with children young people and families. Behavioural and Cognitive Psychotherapy 34, 179-189.

Evans DW, Leckman JF, Carter A, Reznick JS, Henshaw D, King RA, Pauls D (1997). Ritual, habit, and perfectionism: the prevalence and development of compulsive-like behavior in normal young children. Child Development 68, 58-68.

Ferrans CE, Powers MJ (1985). Quality of life index: development and psychometric properties. Advances in Nursing Science 8, 15-24. doi: 10.1097/00012272-198510000-00005

Iniesta-Sepúlveda M, Rosa-Alcázar AI, Sánchez-Meca J, Parada-Navas JL, RosaAlcázar Á (2017). Cognitive-behavioral high parental involvement treatments for pediatric 
obsessive-compulsive disorder: a meta-analysis. Journal of Anxiety Disorders 49, 53-64. doi: 10.1016/j.janxdis.2017.03.010

Kroenke K, Spitzer RL, Williams JB (2001). The PHQ-9: validity of a brief depression severity measure. Journal of General Internal Medicine 16, 606-613.

Lack CW (2012). Obsessive-compulsive disorder: evidence-based treatments and future directions for research. World Journal of Psychiatry 2, 86-90.

Lebowitz ER, Panza KE, Su J, Bloch MH (2012). Family accommodation in obsessive-compulsive disorder. Expert Review of Neurotherapeutics 12, 229-238.

Padesky CA, Mooney KA (1990). Presenting the cognitive model to clients. International Cognitive Therapy Newsletter 6, 13-14.

Peris TS, Bergman RL, Langley A, Chang S, McCracken JT, Piacentini J (2008). Correlates of accommodation of pediatric obsessive-compulsive disorder: parent, child, and family characteristics. Journal of the American Academy of Child and Adolescent Psychiatry 47, 1173-1181.

Rachman S (1998). A cognitive theory of obsessions. In Behavior and Cognitive Therapy Today (ed. E Sanavio), pp. 209-222. Oxford: Pergamon.

Ram D, Kumar A (2014). Compulsive rituals by proxy. Sri Lanka Journal of Psychiatry 5, 29-31.

Rapoport JL, Inoff-Germain G, Weissman MM, Greenwald S, Narrow WE, Jensen PS, Lahey BB, Canino G (2000). Childhood obsessive-compulsive disorder in the NIMH MECA study: parent versus child identification of cases. Methods for the epidemiology of child and adolescent mental disorders. Journal of Anxiety Disorders 14, 535-548.

Salkovskis PM (ed.) (1997). Frontiers of Cognitive Therapy. New York: Guilford Press.

Sheehan V (1983). Sheehan disability scale - overview. Available at: http://memorialparkpsychiatry. com/doc/sheehan_disability_scale.pdf

Spitzer RL, Kroenke K, Williams JB, Löwe B (2006). A brief measure for assessing generalized anxiety disorder: the GAD-7. Archives of Internal Medicine 166, 1092-1097.

Storch EA, Geffken GR, Merlo LJ, Jacob ML, Murphy TK, Goodman WK, Larson MJ, Fernandez M, Grabill K (2007). Family accommodation in pediatric obsessive-compulsive disorder. Journal of Clinical Child and Adolescent Psychology 36, 207-216.

Storch EA, Lehmkuhl H, Pence SL, Geffken GR, Ricketts E, Storch JF, Murphy TK (2009). Parental experiences of having a child with obsessive-compulsive disorder: associations with clinical characteristics and caregiver adjustment. Journal of Child and Family Studies 18, 249-258. doi: 10.1007/s10826-008-9225-y

Thompson-Hollands J, Edson A, Tompson MC, Comer JS (2014). Family involvement in the psychological treatment of obsessive-compulsive disorder: a meta-analysis. Journal of Family Psychology 28, 287-298.

Vikas M, Chandrasekaran R (2011). A case of obsessive-compulsive disorder by proxy. General Hospital Psychiatry 33, 303.

Waters TL, Barrett PM (2000). The role of the family in childhood obsessive-compulsive disorder. Clinical Child and Family Psychology Review 3, 173-184.

\section{Learning objectives}

(1) To better understand the phenomenon of family accommodation in OCD.

(2) To acknowledge the interactive pattern in the case conceptualization of family accommodation in OCD.

(3) To demonstrate the effectiveness of family-based CBT intervention for this condition. 